\title{
Optimisation numérique d'une suspension de véhicule en sollicitation dynamique
}

\author{
Jean-Christophe Walrick ${ }^{1, a}$, SAmir Assaf ${ }^{1}$ et Pascal Longrais ${ }^{2}$ \\ 1 ESTACA, 34-36 rue Victor Hugo, 92300 Levallois-Perret, France \\ 2 ESI GROUP, 99 rue des Solets, SILIC 112, 94513 Rungis Cedex, France
}

Reçu le 25 novembre 2004 ; accepté le 26 mai 2006

\begin{abstract}
Résumé - Le prototypage virtuel joue un rôle important à chaque étape de la conception dans le secteur automobile. Dans ce domaine, le laboratoire de structures et matériaux de l'ESTACA, école d'ingénieur, travaille à la mise en place d'une méthodologie d'analyse mécanique d'une suspension avant de véhicule sous sollicitation dynamique. Cette étude est réalisée en collaboration avec la société ESI-GROUP qui développe et distribue le code Pam-Medysa 2G. Ce travail présente les évolutions successives apportées au modèle de suspension à l'aide de cet outil numérique. À partir d'une première étape de définition de la cinématique du système, différentes améliorations du modèle sont réalisées avec la prise en compte de façon locale du comportement physique (déformation, contraintes dans les composants les plus critiques). Le couplage entre deux approches, l'une globale purement cinématique et l'autre locale par éléments-finis, permet une amélioration du caractère prédictif de la simulation tout en optimisant la durée du calcul explicite dynamique.
\end{abstract}

Mots clés : Suspension double bras / éléments-finis / dynamique / cinématique / angle de carrossage

\begin{abstract}
Numerical optimization for car suspension under dynamic loading. Virtual prototyping plays an important role at every stage of the process design for car manufacturers. Taking this into consideration, ESTACA engineering school carried out a mechanical analysis methodology of a front suspension system. The study is realized in an academic joint project with ESI-GROUP which develops and distributes Pam Medysa 2G software. This work deals with successive improvements of suspension model with the numerical tool. From a first kinematic definition stage, several modifications are made taking into account local physical behavior (stress and strain analysis in the most critical components). By using two numerical models, simulation including global kinematics and local finite element approaches allows an improvement of the predictive feature and reduces the computation cost.
\end{abstract}

Key words: Double arms suspension / finite element / dynamics / kinematics / camber angle

\section{Contexte de l'étude}

Dans la dynamique véhicule, la caractérisation du type de liaison au sol intégrant la suspension et la roue joue un rôle important tant sur le plan du confort des passagers que de la sécurité.

Dans ce contexte, l'étude des caractéristiques mécaniques d'un système multi-corps articulés qui définit une suspension prend une grande importance. Cette suspension absorbe les chocs de la route par son amortisseur mais également par la déformation de la roue et par la déformation élastique des différentes pièces constituantes.

\footnotetext{
a Auteur correspondant : jcwalrick@estaca.fr
}

L'étude porte ici sur une suspension de type train avant à doubles bras. Les principaux avantages de ce type de suspension sont : la possibilité de variation de l'angle de carrossage de la roue, une meilleure stabilité en roulis du véhicule, ainsi qu'une meilleure tenue des amortisseurs [1]. Ce type de suspension est présent sur des véhicules tels que Mercedes classe C-E, Honda Civic 280SE-560SE, Jaguar, Audi A4, A6.

Un tel système est constitué de 5 pièces principales (Fig. 1) : les bras supérieur et inférieur transverses, le porte-moyeu entre ces bras, la tige de direction et la crémaillère. Il comprend 7 joints cinématiques ( 2 pivots, 4 rotules, 1 glissière) soit 27 liaisons géométriques, ce qui donne 2 degrés de liberté pour la roue : mouvement vertical et braquage. 


\section{Nomenclature}

\begin{tabular}{|lll|}
\hline$A$ & $(\mathrm{~m})$ & Amplitude du déplacement sinusoïdal \\
$C$ & $\left(\mathrm{~N} . \mathrm{s} \cdot \mathrm{m}^{-1}\right)$ & Amortissement ressort \\
$f$ & $(\mathrm{~Hz})$ & Fréquence de sollicitation sur route pavée \\
$F$ & $(\mathrm{~N})$ & Force de sollicitation dynamique \\
$K$ & $\left(\mathrm{~N} \cdot \mathrm{m}^{-1}\right)$ & Raideur du ressort d'amortisseur \\
$l_{0}$ & $(\mathrm{~m})$ & Longueur à vide \\
$L e$ & $(\mathrm{~m})$ & Longueur à l'équilibre du ressort \\
$M$ & $(\mathrm{~kg})$ & Masse appliquée \\
$z(t)$ & $(\mathrm{m})$ & Déplacement vertical plateau \\
$\dot{z}(t)$ & $\left(\mathrm{m} . \mathrm{s}^{-1}\right)$ & Vitesse verticale plateau \\
$\ddot{z}(t)$ & $\left(\mathrm{m} . \mathrm{s}^{-2}\right)$ & Accélération verticale du plateau \\
$\varphi$ & $(\mathrm{Rad})$ & Déphasage pour sollicitation sinusoïdale en déplacement \\
\hline
\end{tabular}
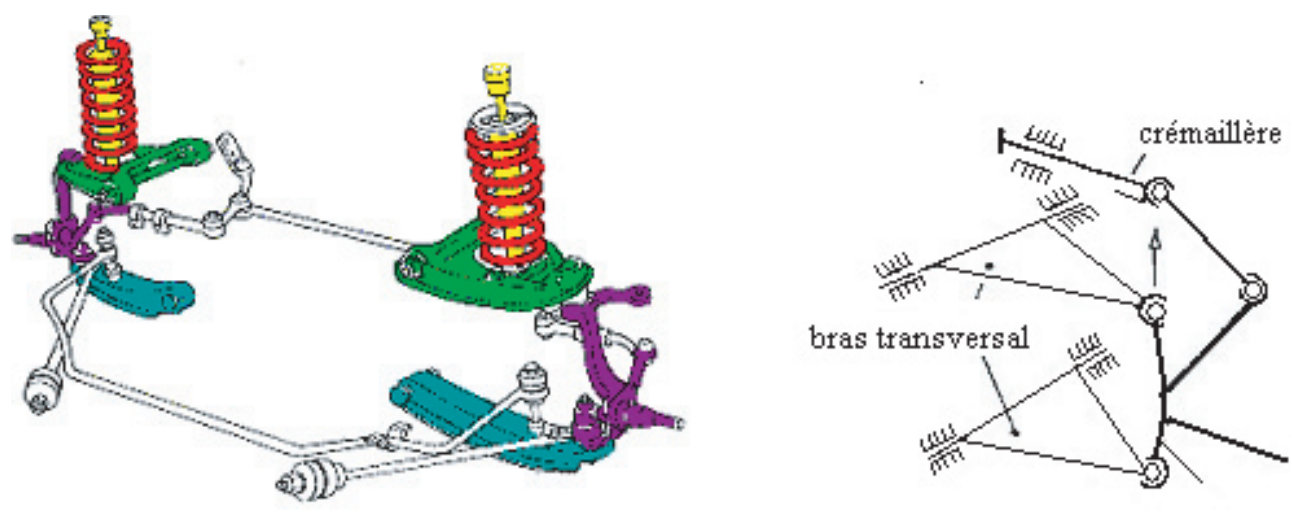

Fig. 1. Descriptif cinématique de la suspension adoptée pour l'étude.

Parmi les critères dimensionnant la liaison, les sollicitations dynamiques causées par la configuration de la route (choc, vibrations) jouent un rôle important. La plupart des modèles réalisés se concentrent principalement sur les caractéristiques cinématiques en considérant les différents constituants comme des corps rigides. Certaines études existent incluant un modèle élasto-cinématique des joints constituant les liaisons de la suspension [2].

Cette approche a pour but de définir une modélisation qui couple un modèle cinématique avec une simulation qui intègre les caractéristiques physiques de certains des composants les plus sollicités. Le logiciel Pam-Medysa permet ce type d'étude par une première simulation purement cinématique du système réalisée grâce au module Motion intégré. L'action du sol sur le système suspension et roue est définie en terme de sollicitation dynamique en déplacement. Une seconde étape via le module Medysa Editor permet d'inclure, grâce à une modélisation éléments-finis, la physique de certains composants critiques tels le porte-moyeu entre les bras et le pneu dont la déformation joue un rôle important.

Cet article présente différentes simulations et les améliorations successives du système en terme de géométrie et de caractéristiques afin de se rapprocher des critères constructeurs en terme de masse et de critères mécaniques [3].

\section{Définition du modèle initial}

\subsection{Mise en place des caractéristiques cinématiques et validation}

Une première définition géométrique des différents composants est réalisée à l'aide du logiciel CATIA d'après un modèle standard.

Les composants sont alors importés sous le module MOTION-EDITOR intégré à Pam_Medysa et le modèle cinématique est créé (Fig. 2a) :

- Trois liaisons-rotules réparties entre le porte-moyeu central et les bras supérieur et inférieur, ainsi qu'avec la biellette de direction.

- Une liaison-rotule entre la biellette de direction et la crémaillère.

- Trois liaisons-pivots réparties entre les axes des bras supérieur et inférieur et le châssis, ainsi que l'axe du moyeu et la roue.

- Deux liaisons-glissières, l'une entre la crémaillère et le châssis, l'autre à la base du plateau.

- Un ressort d'amortissement entre le bras supérieur et le châssis, représenté par une pièce cubique fixe.

L'un des points importants est la définition des caractéristiques mécaniques du ressort sous MOTION. 


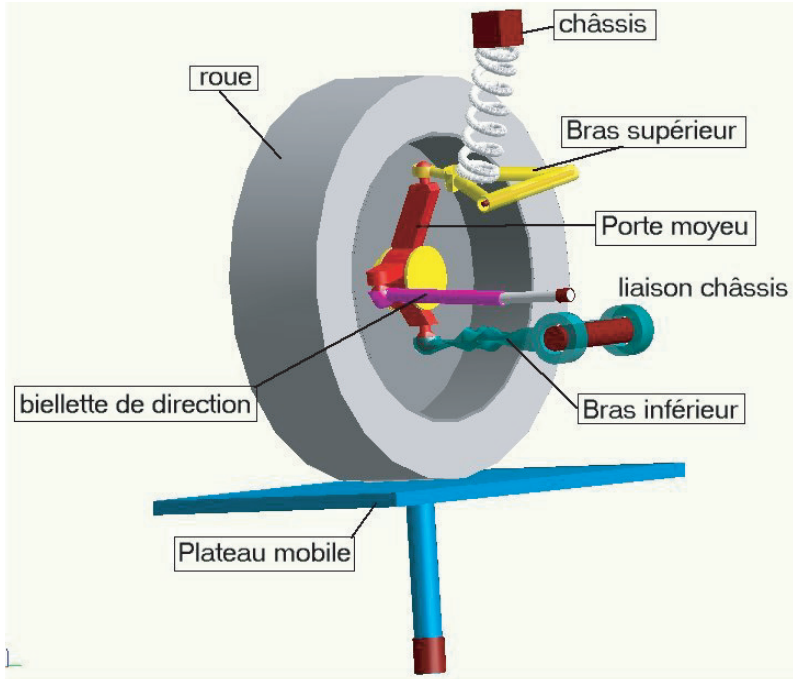

(a)

\section{Force (N)}

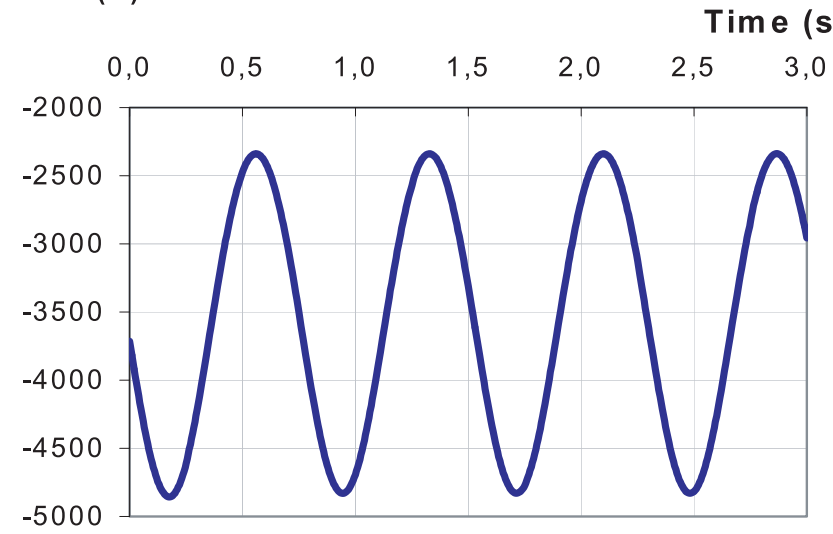

(b)

Fig. 2. Modèle cinématique initial sous MOTION.

Sous sollicitation dynamique du ressort, la loi de Newton donne :

$$
M . \ddot{z}(t)+C . \dot{z}(t)+K . z(t)=F
$$

Pour ce faire, on considère que $66 \%$ de la masse du véhicule est répartie sur les suspensions avant, soit pour cette étude $800 \mathrm{~kg}$, donc $400 \mathrm{~kg}$ sur chaque suspension.

Pour une telle suspension, la première fréquence propre du système est donnée à $1,3 \mathrm{~Hz}$ [4], soit :

$$
f=\frac{1}{2 \pi} \sqrt{\frac{K}{M}}
$$

Ce qui permet de donner une estimation de la raideur du ressort telle que :

$$
\begin{aligned}
K=(2 \pi f)^{2} \cdot M=(2 \pi \cdot 1,3)^{2} & \cdot 400 \\
K & =26660 \mathrm{~N} . m^{-1}
\end{aligned}
$$

Ainsi, la longueur à vide du ressort est définie pour respecter l'équilibre statique avec la masse du véhicule.

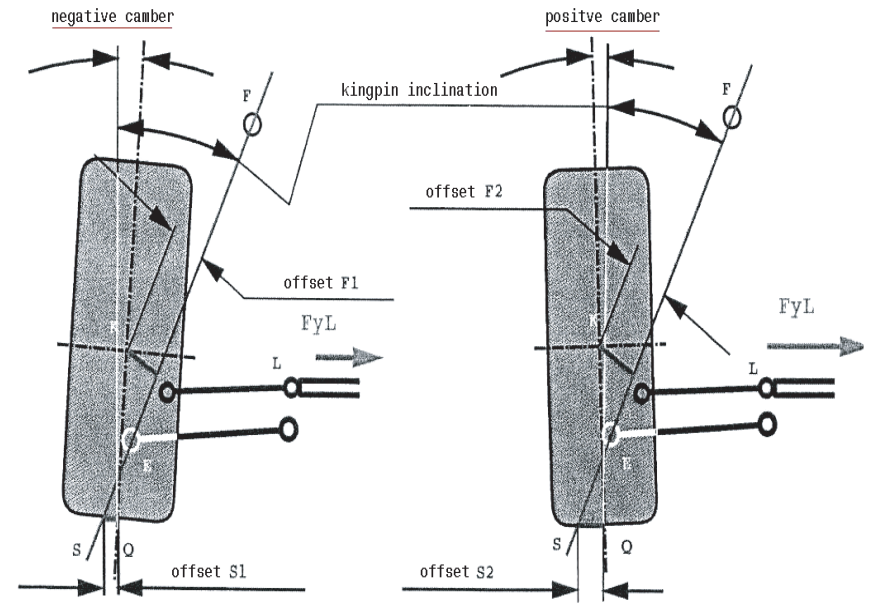

(a)

Camber angle

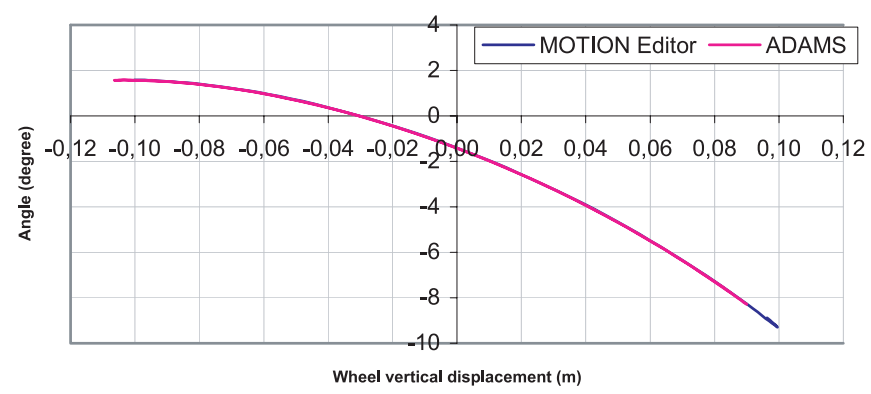

(b)

Fig. 3. Variation de l'angle de carrossage pris au niveau de la roue.

Longueur du ressort à l'équilibre statique : $l_{\mathrm{e}}=$ $0,200 \mathrm{~m}$

Longueur à vide du ressort $l_{0}=0,333 \mathrm{~m}$

Pour la première simulation un amortissement faible est appliqué au ressort afin de vérifier la fiabilité du modèle cinématique sur une plus grande amplitude en déplacement $\left(C=460\right.$ N.s.m $\left.{ }^{-1}\right)$

Une première simulation cinématique est réalisée sous MOTION pour le système suspension et roue d'environ $18 \mathrm{~kg}$. Outre les joints cinématiques du système décrit précédemment, un joint type plan-disque $2 \mathrm{D}$ est défini entre la roue et le plateau qui représente la route.

Une sollicitation en déplacement vertical de la route est assurée via une liaison-glissière en base du plateau. Une excitation périodique sinusoïdale est définie pour le déplacement du plateau d'amplitude 0,1 m et de fréquence $1,3 \mathrm{~Hz}$.

La courbe de la figure $2 \mathrm{~b}$ montre l'évolution de la force de réaction du ressort autour de sa configuration d'équilibre statique préalablement définie.

L'inclinaison de la roue autour de l'axe vertical définit l'angle de carrossage (Fig. 3a). C'est l'un des paramètres qui permet de définir le comportement cinématique de la liaison. La figure 3b présente l'évolution de cet angle de carrossage mesuré en fonction du déplacement vertical. 


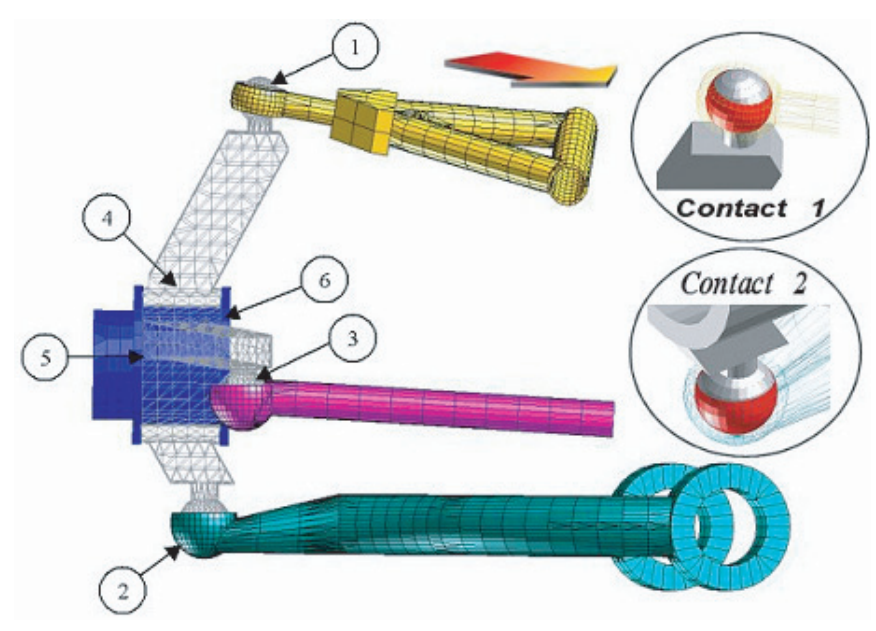

Fig. 4. Localisation des contacts éléments-finis autour du porte-moyeu.

Cette courbe est caractéristique d'une suspension avant double bras. Afin de disposer d'une base de validation cinématique, ces résultats ont été comparés à ceux obtenus avec un modèle réalisé sous le logiciel cinématique multi-corps ADAMS de MSC-Software. On constate alors, que les courbes des deux modèles sont parfaitement superposables.

\subsection{Caractéristiques du modèle éléments-finis associé}

Au calcul cinématique précédent, un modèle élémentsfinis est associé afin d'inclure localement la physique de matériaux (concentration de contraintes, déformation) dans le porte-moyeu.

Lors de cette modélisation initiale, la déformation du pneu a été négligée en première approximation. L'étude physique de la déformation s'est portée sur ce porte-moyeu, pièce de transmission centrale, soumise aux actions de l'ensemble des autres composants.

Le porte-moyeu déformable est maillé sous le module GEOMESH de Pam-Medysa à l'aide d'éléments volumiques tétraédriques. Cette nouvelle modélisation du porte-moyeu remplacera la pièce rigide correspondante dans la simulation cinématique. Cela rend caducs les joints cinématiques précédemment définis entre ce portemoyeu et les pièces en liaison. Il est alors nécessaire de définir une gestion des contacts par pénalité de type éléments-finis. Pour cela, des ensembles de nœuds esclaves sont définis sur la pièce déformable, qui subissent les efforts transmis des pièces rigides périphériques via les surfaces maîtres. Ceci implique le maillage surfacique des pièces rigides afin de définir les surfaces en contact, soit 6 contacts différents (Fig. 4).

La mise en donnée éléments-finis comprenant les caractéristiques matériau de l'aluminium avec une loi élastique-plastique pour le porte-moyeu, gestion de contact, sont définis sous le module Medysa-Editor.

Concernant les autres pièces maillées en surfacique, elles restent rigides et le matériau spécifique associé permet la gestion des contacts. Les autres pièces

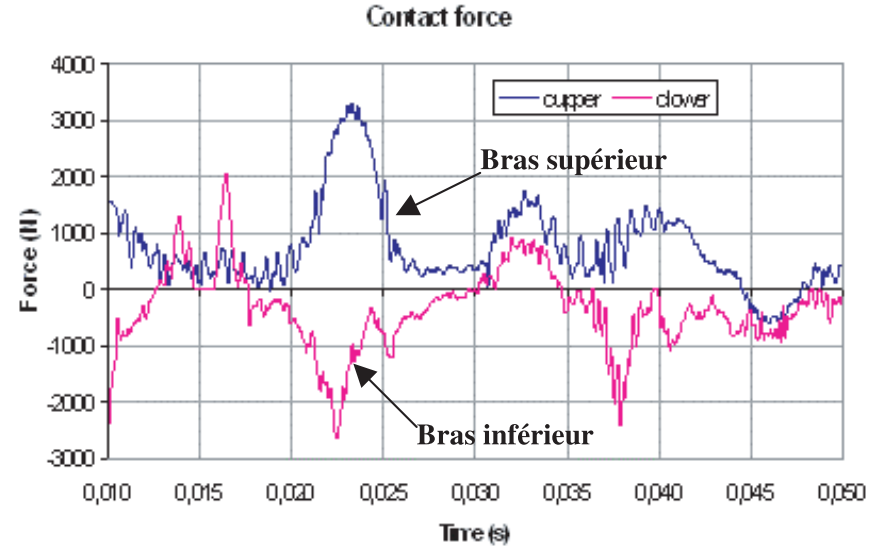

Fig. 5. Résultats issus du couplage des modèles cinématique et éléments-finis.

non-périphériques au porte-moyeu (roue, plateau) sont maillées uniquement pour pouvoir être visualisées en posttraitement du calcul éléments-finis.

La nouvelle simulation effectuée couple le calcul cinématique global précédemment réalisé et le calcul éléments-finis défini. Le calcul cinématique pilote les sollicitations en déplacements imposées, l'action de la roue rigide par rapport au plateau, ainsi que les liaisons au niveau du châssis fixe (pivots en extrémité des bras et ressort entre le bras supérieur et le châssis). Dans ce calcul cinématique le porte-moyeu rigide est éliminé et son influence sur le système est restituée par son modèle éléments-finis déformable. Ainsi les sollicitations cinématiques sont appliquées, via les contacts élémentsfinis, au maillage du porte-moyeu déformable. Les efforts internes à cette pièce sont restitués sous forme de réactions appliquées aux pièces périphériques dans le calcul cinématique. Ainsi l'interaction entre les deux modèles s'opère tout au long du calcul couplé.

\subsection{Résultats issus du calcul couplé}

Les résultats de ce couplage de deux calculs peuvent permettre de considérer l'influence du comportement physique du porte-moyeu face au calcul purement cinématique, sur un temps de $0,05 \mathrm{~s}$.

Les forces de contact aux rotules des bras supérieure et inférieure évoluent en traction-compression avec une valeur maxi de $3000 \mathrm{~N}$ (Fig. 5).

L'évolution de la déformation globale longitudinale du porte-moyeu, sur un cycle de déplacement du sol, reste inférieure à $0,7 \%$ (Fig. 6).

Une comparaison de l'évolution de l'angle de carrossage sur un cycle entre une simulation cinématique et la simulation couplée éléments-finis présente de grandes similitudes (Fig. 7).

Dans cette première partie, une validation de la méthodologie a été réalisée pour un système multi-corps avec prise en compte des déformations du porte-moyeu. 
(\%)

Déformation longitudinale

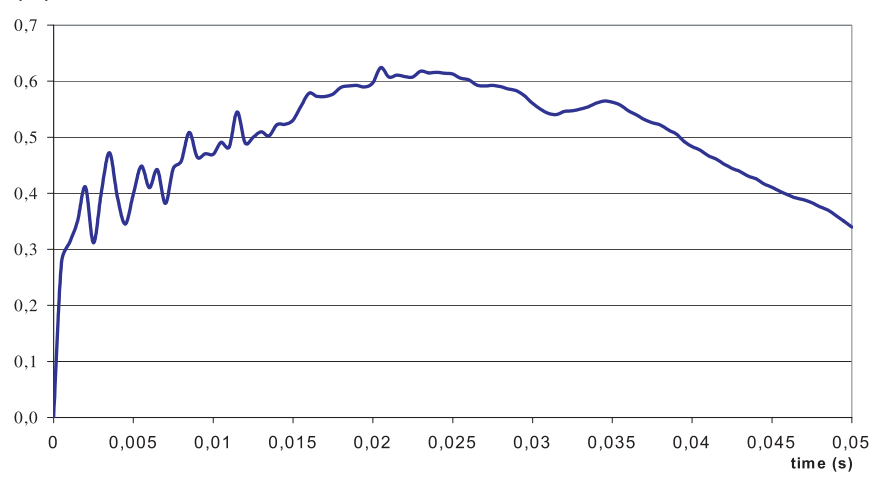

Fig. 6. Évolution de la déformation longitudinale du portemoyeu.

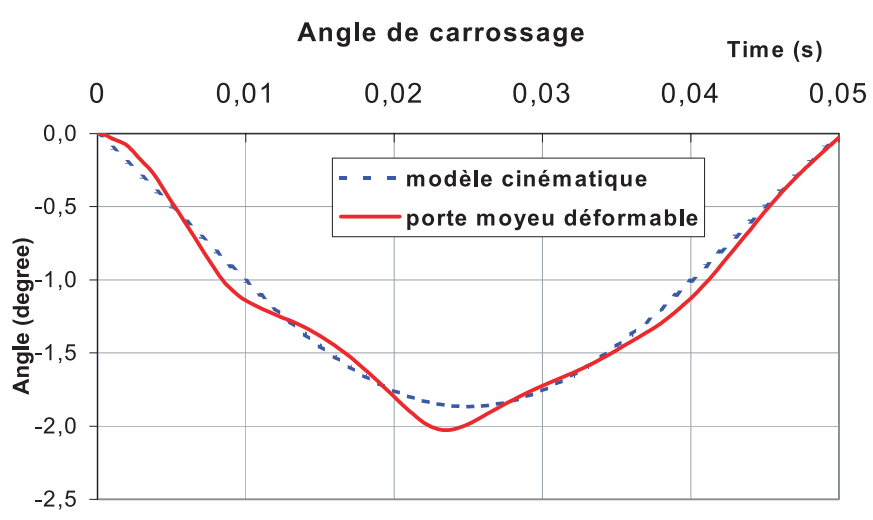

Fig. 7. Comparaison d'évolution de l'angle de carrossage.

Cependant ce modèle montre des limites :

- Certaines pièces, notamment le porte-moyeu apparaissent assez massives, ce qui ne favorise pas de déformation des pièces.

- Le châssis auquel sont rattachés les bras supérieur et inférieur ainsi que le ressort est considéré fixe, or le mouvement transmis au châssis est un paramètre important dans la caractérisation d'une suspension.

- Il ne prend pas en considération la déformation du pneumatique qui joue un rôle important dans l'amortissement des chocs.

\section{Optimisation du modèle de liaison au sol}

\subsection{Caractéristiques du modèle cinématique}

Suite aux conclusions de l'étude précédente, le modèle a été optimisé en plusieurs campagnes de simulations : différentes pièces telles le porte-moyeu, la jante, la crémaillère ont été redéfinies à l'aide du logiciel CATIA, l'objectif étant d'avoir des pièces optimisées en forme et en masse. Ainsi sous MOTION, la masse du porte-moyeu passe de 2,46 kg à 1,66 kg pour une même densité de matériau. Par souci de réalisme le pneu a lui aussi été redessiné.
Un composant additionnel représentant le châssis est connecté au système précédemment défini via les liaisons pivot des deux bras et le ressort (Fig. 8). Une liaison glissière supplémentaire permet de prendre en compte le mouvement vertical de ce châssis induit par la route et transmis par la suspension. La densité du composant est choisie afin de respecter une masse de $400 \mathrm{~kg}$.

Concernant la simulation élément-finis couplée, le pneu maillé peut être considéré déformable grâce à un modèle d'airbag développé pour les études de crash. Ce modèle assez complet prend en compte les caractéristiques thermodynamiques (pression, volume) ainsi que la vitesse de gonflage à stabilisation, ce qui permet de contrôler l'amortissement du pneumatique. Ce modèle est compris dans une structure membranaire au sein d'un maillage volumique du pneu.

\subsection{Modélisation d'un passage sur route pavée}

Avant toute sollicitation dynamique, l'équilibre statique sous MOTION est vérifié en s'assurant que la position du châssis reste constante dans le temps si le plateau est statique.

L'excitation de type passage sur route pavée choisie est prise similaire à une sinusoïde, dont l'amplitude et la fréquence de déplacement vertical du plateau sont paramétrables, soit :

$$
z(t)=A \cdot \sin (2 . \pi \cdot f \cdot t+\varphi)
$$

Concernant l'amplitude, le choix s'est fixé sur $1 \mathrm{~cm}(A=$ $0,01 \mathrm{~m})$.

Un déphasage de $\varphi=\pi / 2$ est choisi afin d'éviter d'avoir une vitesse instantanée à $t=0 \mathrm{~s}$.

Pour la fréquence, diverses simulations cinématiques ont été effectuées sur une durée de $3 \mathrm{~s}$, en faisant varier la fréquence de 2 à $55 \mathrm{~Hz}$. L'amplitude de déplacement du châssis mobile sur cette durée est reporté sur le graphe figure 9 .

Les courbes relatives aux 4 fréquences montrent une tendance commune de stabilisation dynamique du châssis autour d'une position d'équilibre après une phase transitoire de l'ordre de la seconde. L'amplitude d'oscillation est inversement proportionnelle à la fréquence d'excitation.

La position moyenne de stabilisation du châssis évolue avec une fréquence plus élevée de $55 \mathrm{~Hz}$, due aux effets d'inertie. Cette tendance est vérifiée avec l'augmentation de la fréquence.

En raison du couplage avec des modèles déformables éléments-finis, il paraît utile, pour séparer les effets, d'éviter les fréquences de sollicitation trop faibles donnant une forte amplitude d'oscillation au châssis. Aussi, une fréquence de sollicitations $f=55 \mathrm{~Hz}$ est choisie, ce qui correspond au passage d'une voiture à $50 \mathrm{~km} \cdot \mathrm{h}^{-1}$ sur des pavés de $25 \mathrm{~cm}$ de long.

Suite à la définition du modèle cinématique, deux modèles éléments-finis correspondants peuvent être réalisés : l'un avec le pneu déformable (Fig. 10a), l'autre 


\section{Assemblage}

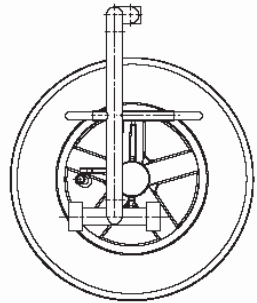

Vue de droite

$[1$

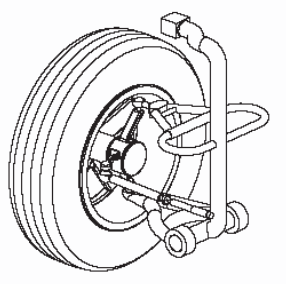

Vue isométrique

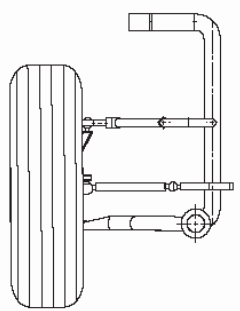
Vue de face

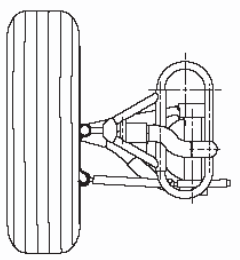

Vue de dessus
Echelle : $1: 8$

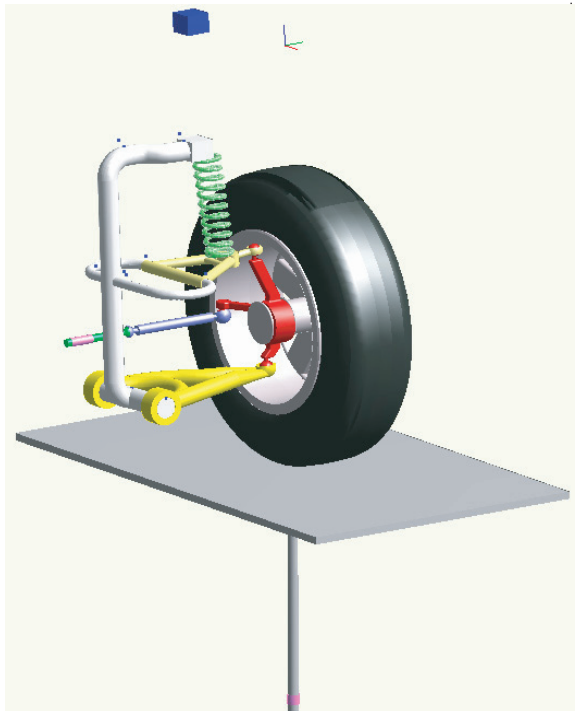

Modèle cinématique réalisé

Fig. 8. Caractère du nouveau système de suspension modélisé.

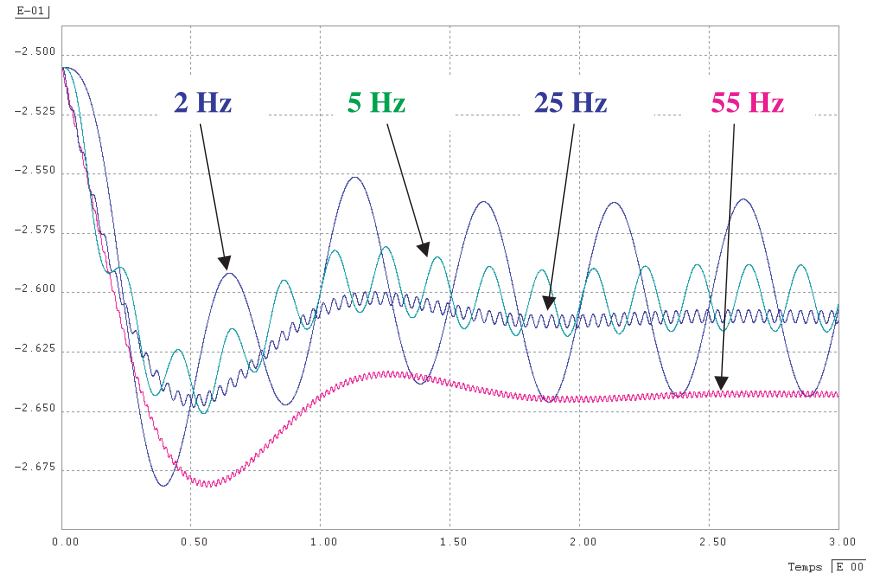

Fig. 9. Évolutions en déplacement du châssis pour la modélisation cinématique.

avec le porte-moyeu déformable et pneu rigide. La figure 10b montre que le châssis en phase transitoire s'enfonce de $3,8 \mathrm{~cm}$ au lieu de $1,7 \mathrm{~cm}$ pour le modèle cinématique et reste en accord avec la déformation du pneu. Cette différence est moindre pour le cas où seule porte-moyeu est déformable (Fig. 11a) qui reste assez proche du modèle cinématique (Fig. 11b). Ces observations sont en accord avec les amplitudes de variation de longueur du ressort à chaque oscillation qui est de l'ordre de $15 \mathrm{~mm}$ pour la simulation cinématique, $13 \mathrm{~mm}$ avec le seul porte-moyeu déformable et $2 \mathrm{~mm}$ avec la seule roue déformable.

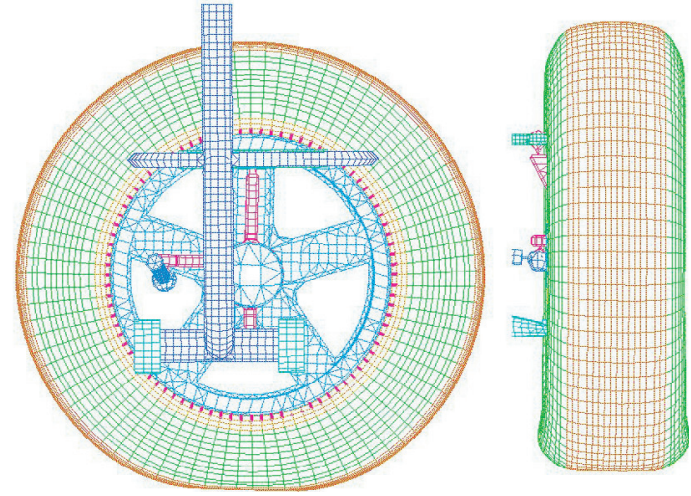

(a)

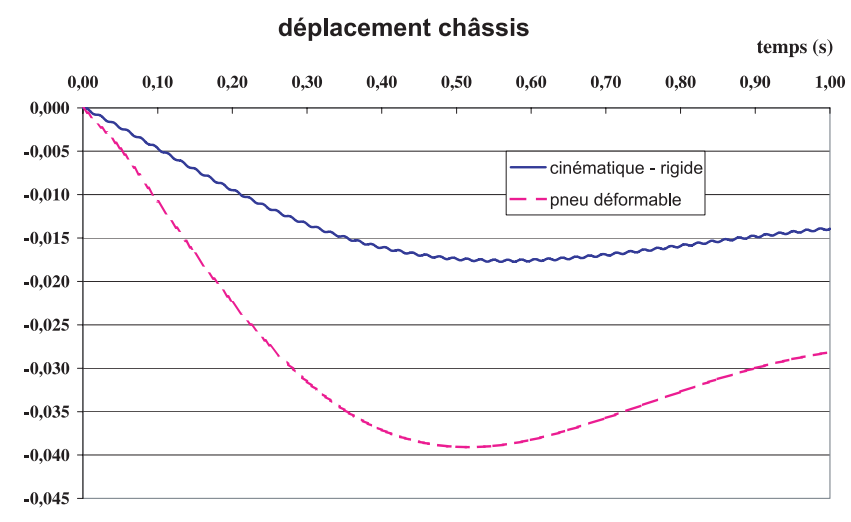

(b)

Fig. 10. Simulation passage d'une route pavée avec pneu déformable. 


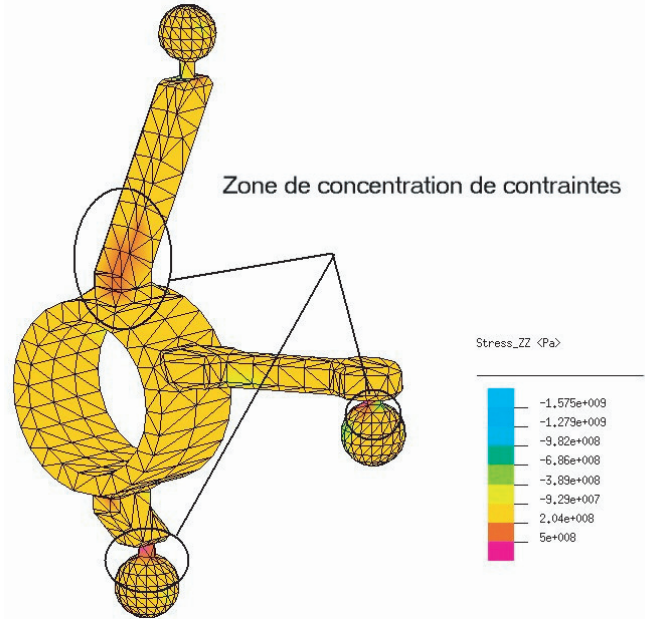

(a)

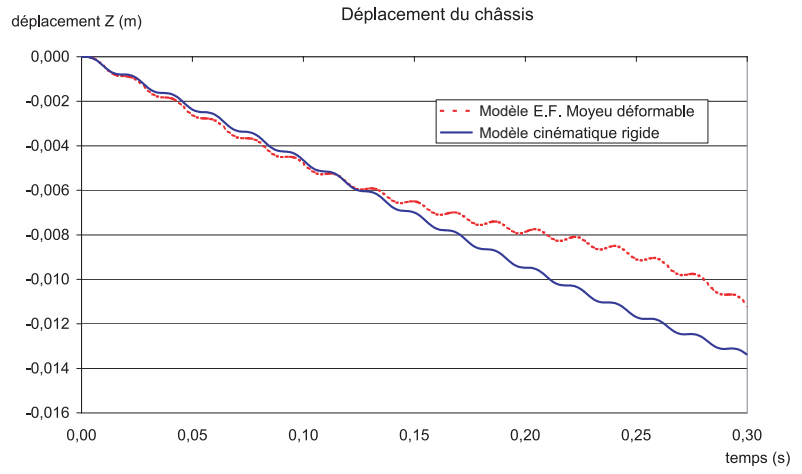

(b)

Fig. 11. Simulation éléments-finis avec porte-moyeu déformable.
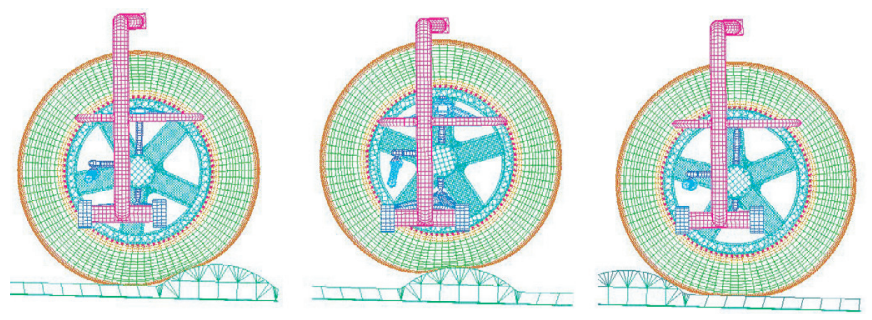

Fig. 12. Passage de la bosse par un modèle pneu déformable.

\subsection{Modélisation d'un passage « dos d'âne »}

Cette modélisation simule le passage d'une bosse par le système avec la roue modélisée comme précédemment. La simulation correspond au passage d'une bosse de $60 \mathrm{~mm}$ de hauteur à $4,5 \mathrm{~m} . \mathrm{s}^{-1}$.

Différents instants du passage d'une bosse sont présentés (Fig. 12) et les déplacements associés au niveau du châssis et à la base du moyeu rigide sont enregistrés (Fig. 13).

Les résultats montrent une configuration de déformation du pneu lors du passage de la bosse tout à fait acceptable : il n'y a pas de décollement du pneu avec la route et de rebond excessif. Le châssis

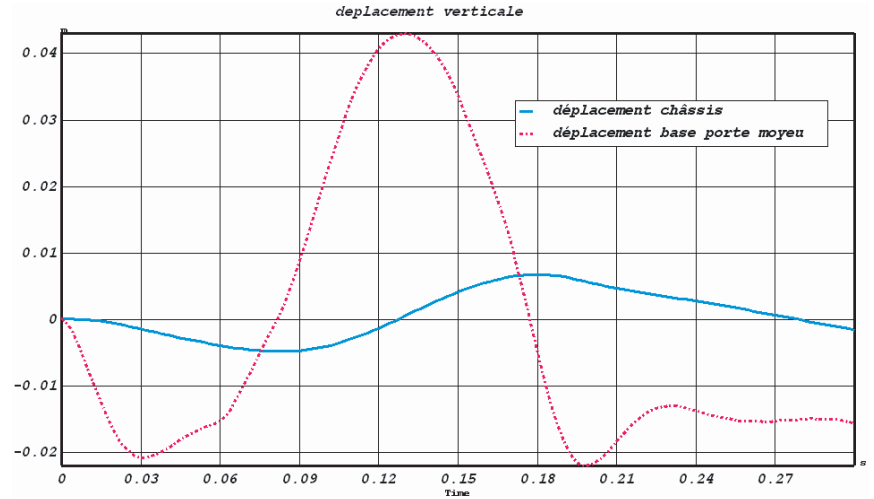

Fig. 13. Déplacement vertical du châssis et en base de portemoyeu.

se déplace sur une faible amplitude au regard du déplacement $\mathrm{du}$ porte-moyeu, ce qui caractérise un amortissement important du système lors du passage de la bosse.

\section{Conclusion}

L'objet de cette étude est de déterminer l'influence de pièces déformables (pneu et porte-moyeu) sur le comportement global d'une suspension avant double bras. L'étude réalisée via le logiciel Pam Medysa couple un modèle cinématique corps rigides et un modèle élémentsfinis.

La première étape a été de réaliser un premier modèle cinématique et de valider sa réponse globale. Un modèle éléments-finis de porte-moyeu déformable a été couplé au modèle cinématique et validé par l'évolution des contraintes et déformations localisées. Suite aux résultats de cette étude des améliorations ont été réalisées sur cette liaison : les géométries de certaines pièces porte-moyeu, jante, pneu ont été optimisées. De même le mouvement du châssis est pris en compte et un modèle éléments-finis de pneu déformable est intégré.

Les simulations sous sollicitations dynamiques de passage de pavés et de bosses effectuées ont permis de montrer l'importance de la prise en compte de la déformation du pneumatique vis-à-vis du comportement général de la suspension. L'influence de la seule déformation du portemoyeu semble moindre sur le comportement global.

L'avantage d'un tel couplage est de pouvoir optimiser le temps de calcul dans la simulation d'un modèle multi-corps sous sollicitation dynamique au regard d'une simulation complète par éléments-finis en calcul explicite.

Les perspectives de ce travail sont nombreuses : outre le dimensionnement des pièces en choc [5], une campagne de simulations en vue d'un dimensionnement en fatigue peut être envisagée. De même la prise en compte des efforts externes tels que l'influence de la biellette de direction sur le braquage et les contraintes amenées par la réaction de la route via le pneu [6] seraient nécessaires. 
De plus, la compatibilité de la version 2004 de Pam-medysa avec Motion CATIA (Solid-dynamics) permettra une définition cinématique directe du système sous CATIA V5, pour une utilisation sous Medysa.

\section{Références}

[1] T.D. Gillespie, Fundamentals of vehicle dynamics, Society of Automotive Engineers Inc., 1992

[2] M.M. Hiller, C. Woernle, Elasto-kinematical analysis of five-points wheel suspension, ingénieurs de l'automobile, October 1985, pp. $77-80$

[3] M.F. Renard, M.A. Samy, Conception et optimisation d'un système de suspension automobile. Automobile suspension design and optimization, Congrès International S.I.A.,
Apport du calcul scientifique en conception automobile, École Polytechnique de Palaiseau, 1993

[4] P. Barak, Effects of suspension design factors on ride and handling in the design cycle of passenger car and light trucks, Congrès International S.I.A., La dynamique du véhicule automobile et ferroviaire, École Centrale de Lyon, 1997

[5] M.M.A. Tranzer, G. Berthias, Calcul au choc des pièces mécaniques de liaison au sol - exemple des roues en alliage léger - Impact simulation of mechanical components of suspension devices - Example of light alloy wheels, Congrès International S.I.A., Apport du calcul scientifique en conception automobile, École Polytechnique de Palaiseau, 1993

[6] E. Bakker, L. Nybor, B.B. Pacejka, Tyre modelling for use in vehicle dynamics studies, Society of Automotive Engineers, 1988 